\title{
SUPERCONDUCTING MAGNETS FOR INDUCTION LINAC PHASE-ROTATION IN A NEUTRINO FACTORY
}

\author{
M. A. Green, and S. Yu \\ Lawrence Berkeley National Laboratory, Berkeley CA 94720, USA
}

\begin{abstract}
The neutrino factory[1-3] consists of a target section where pions are produced and captured in a solenoidal magnetic field. Pions in a range of energies from $100 \mathrm{Mev}$ to 400 $\mathrm{MeV}$ decay into muons in an 18-meter long channel of $1.25 \mathrm{~T}$ superconducting solenoids. The warm bore diameter of these solenoids is about $600 \mathrm{~mm}$. The phase rotation section slows down the high-energy muon and speeds up the low energy muons to an average momentum of $200 \mathrm{MeV} / \mathrm{c}$. The phase-rotation channel consists of three induction linac channels with a short cooling section and a magnetic flux reversal section between between the first and second induction linacs and a drift space between the second and third induction linacs. The length of the phase rotation channel will be about 320 meters. The superconducting coils in the channel are $0.36 \mathrm{~m}$ long with a gap of $0.14 \mathrm{~m}$ between the coils. The magnetic induction within the channel will be 1.25 . For 260 meters of the 320-meter long channel, the solenoids are inside the induction linac. This paper discusses the design parameters for the superconducting solenoids in the neutrino factory phase-rotation channel.
\end{abstract}

\section{THE TYPES OF SUPERCONDUCTING SOLENOIDS IN THE PHASE- ROTATION AND MINI-COOLING CHANNEL}

The first induction linac, the min-cooler, the second and third induction linacs extend from $320 \mathrm{~m}$ from the pion decay section downstream from the target to the muon bunching and cooling section. Within this phase rotation region, there are four types of solenoids[3,4]. They are 1) There is 18 -meters of $1.25 \mathrm{~T}$ decay channel solenoids. There are six of these magnets with a $800 \mathrm{~mm}$ warm bore. The $100-\mathrm{mm}$ space between the 600 $\mathrm{mm}$ beam bore and the cryostat warm bore is filled by a water-cooled copper shield. 2) The first induction linac solenoids, which extend $110 \mathrm{~m}$ from the pion decay section. Around the $600-\mathrm{mm}$ diameter beam bore is a $10-\mathrm{mm}$ thick water-cooled copper radiation shield. The warm bore of this cryostat is $620 \mathrm{~mm}$. There are one hundred-ten magnets of this type. 3) The second and third induction linacs and the drift between them extend $190 \mathrm{~m}$ to the start of the bunching system. These solenoids have no radiation shield and have a cryostat warm bore diameter of $600 \mathrm{~mm}$. There are one hundred-ninety magnets of this type. 4) There are 
two solenoid sections that surround the hydrogen absorber for the mini-cooler. These solenoids will have a warm bore diameter of $800 \mathrm{~mm}$. There are six of these magnets. 5) There is a single field flip solenoid between the two min-cooling sections is 2.0-meters long, with a warm bore diameter of $400 \mathrm{~mm}$. Table 1 shows the design parameters for the two types induction linac solenoids and 12 large bore solenoids. The 2-meter long field flip solenoid is not included in Table 1. Figure 1 shows a cross-section of the induction cell and mini-cooling solenoids. 
TABLE 1. Decay, Phase Rotation, and Mini-cool Solenoid Parameters

Number of Cells of This Type

Cell Length (mm)

Magnet Cryostat Length (mm)

Number of Coils per Magnet Cryostat

Length of Each S/C Coil (mm)

Space Between the S/C Coil (mm)

Inner Cryostat Radius (mm)

S/C Coil Inner Radius (mm)

Magnet Cryostat Thickness at Ends (mm)

\section{Magnets}

110

1000.0

900.0

2

360.0

140.0

310.0

334.0

55.0

207.6

277.3

1.25

$\pm 2.5$

$\sim 1.6$

2532

392.8

4.4

$\sim 1600$

224

2.90

249

$1.39 \times 10^{22}$

quench-back
Later Induction

Magnets

Large Bore

Magnets

12

3000.0

2900.0

6

360.0

140.0

400.0

429.0

80.0

911.1

1151.1

1.25

$\pm 2.2$

$\sim 1.6$

7596

392.8

4.4

$\sim 1600$

1103

14.3

249

249

$1.31 \times 10^{22}$

$6.86 \times 10^{22}$

quench-back quench-back

Quench Protection Method

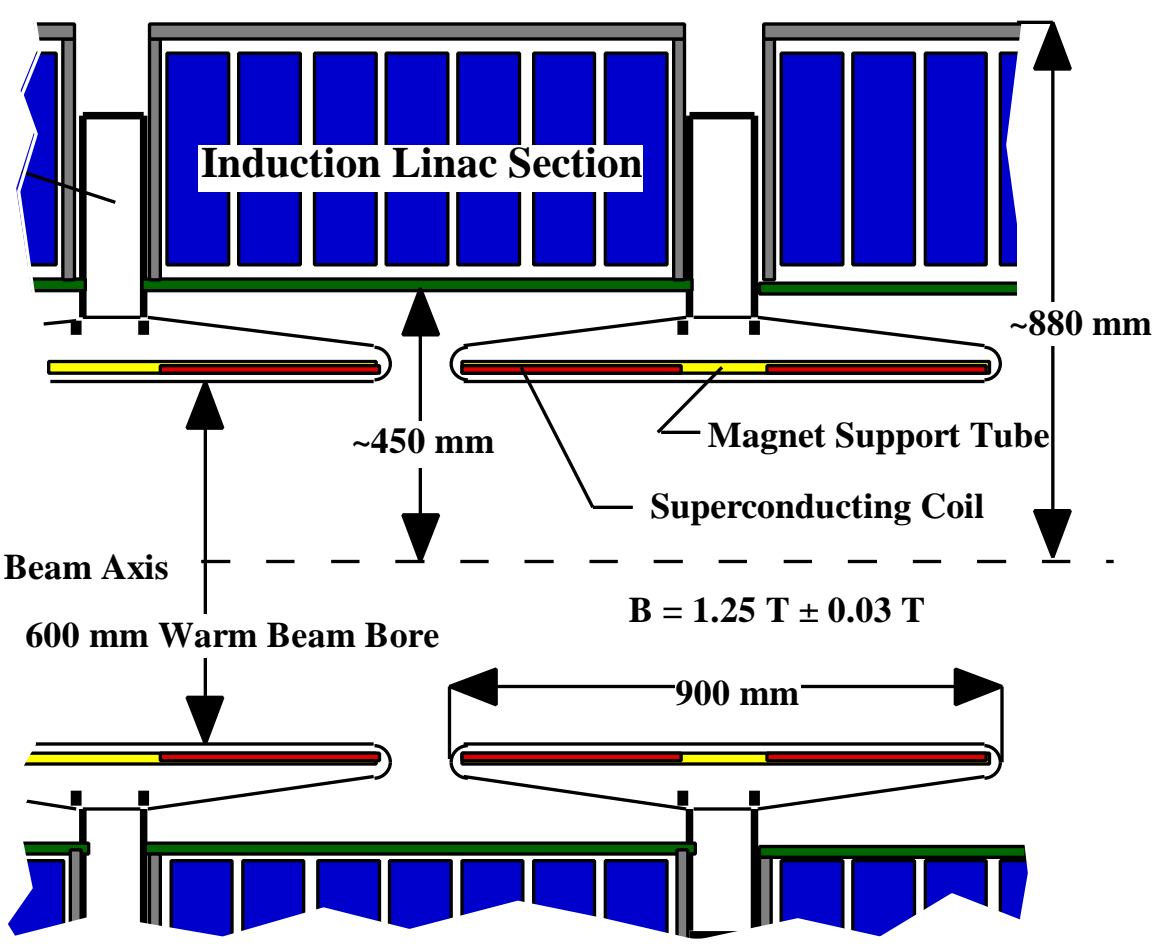


FIGURE 1. A Cross-section of the Solenoid in an Induction Cell downstream from the Mini-cooler

\section{PHASE-ROTATION INDUCTION LINAC THIN SOLENOIDS}

The basic requirements for the phase rotation linac are as follows[5]: 1) The magnetic induction in the phase-rotation and mini-cooling channel has been set to $1.25 \mathrm{~T}$. This means that the bore diameter for the beam is $600 \mathrm{~mm}$. 2) The period of the varying magnetic field on the axis of the phase-rotation channel should be minimized. This means that the coils in the cell should be of equal length with equal length gaps between the coils. A 1.0-meter long cell can have two equal length coils coil and two equal length spaces between coils, which yields a period length for the magnetic field of 0.5 meters. 3) The radial thickness of the solenoid cryostat should also be minimized. This allows the induction linac structure to be brought closer to the axis of the machine. A second factor that influences the distance the induction cell can be from the magnetic field axis is the magnetic flux leakage through the gaps between the superconducting coils. 4) The space between the induction linac cells must be minimized. This means that the space used for the cold mass support system, the electrical leads and the cryogen feed system must fit in this minimum space. 5) Field correction dipoles are assumed to be mounted on the inner bore of the solenoid coils. The pair of dipoles is about 1.0-mm thick and they will correct alignment errors up to $5 \mathrm{mrad}$.

Figure 2 shows a cross-section of a typical superconducting solenoid that is designed to generate an average induction of $1.25 \mathrm{~T}$ on the axis of the phase-rotation induction linac. The inner bore radius of the solenoid cryostat is $300 \mathrm{~mm}$. This allows a $200 \mathrm{MeV}$ muon beam with a nominal diameter of $600 \mathrm{~mm}$ to pass through the solenoid without loss (except from muon decay). The distance from the end of the superconducting coil to the outside end of the cryostat was reduced to $20 \mathrm{~mm}$. If an additional support clip is needed at the end of the coil, the coils can be shortened to accommodate the clip in the space shown. The coils in the solenoid shown in Figure 1 have a length of $360 \mathrm{~mm}$. The gap between the coils is $140 \mathrm{~mm}$ and the space between a coil in one magnet and the coil in the next magnet is also $140 \mathrm{~mm}$. 


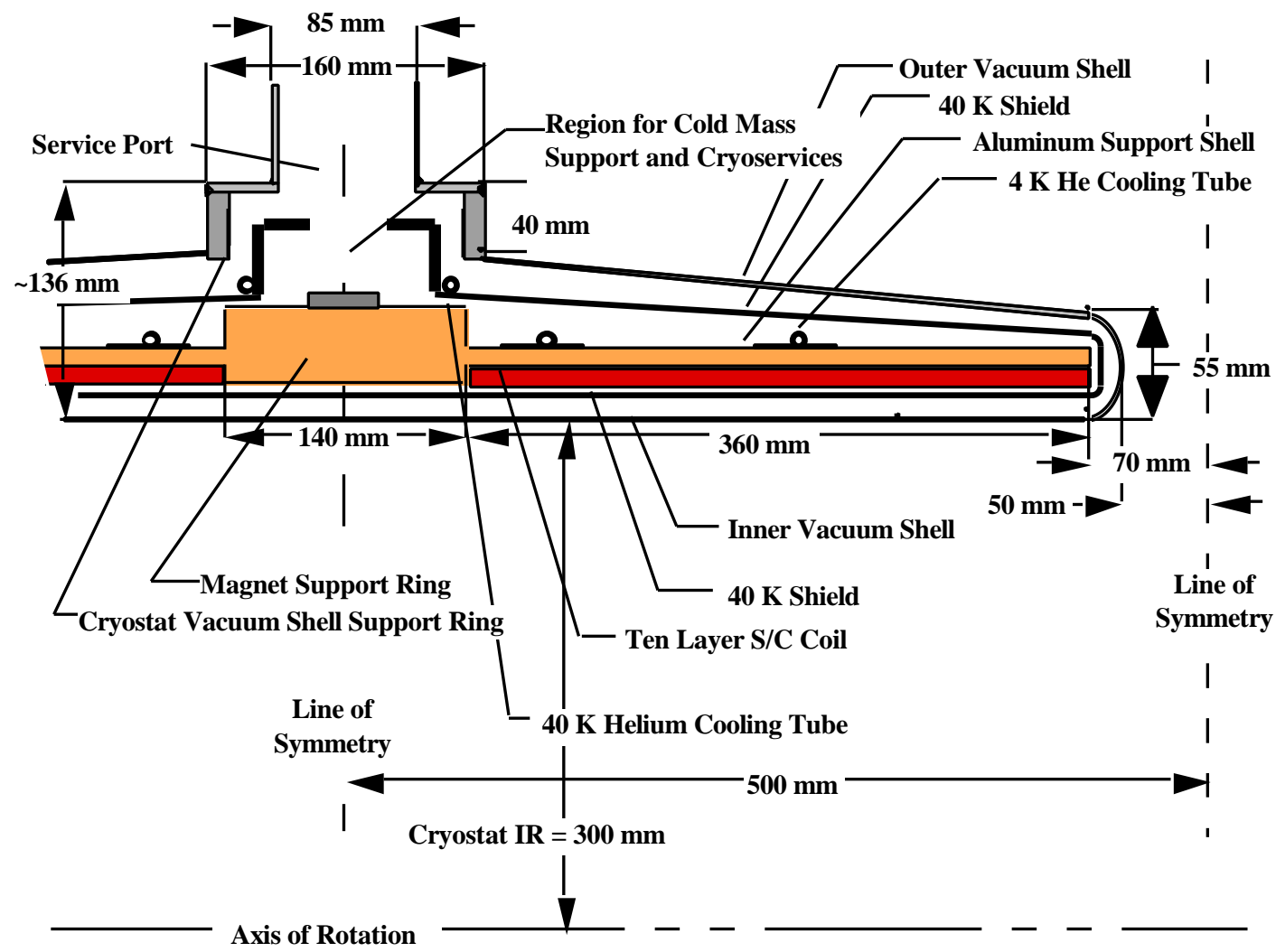

FIGURE 2. A cross-section of a Typical Phase Rotation Induction Cell Superconducting Coil and Cryostat 
The proposed conductor for coils shown in Figure 2 is a standard MRI magnet conductor that is 1 part $\mathrm{Nb}-\mathrm{Ti}$ and 4 parts $\mathrm{RRR}=70$ copper. This conductor has fifty-five $85-\mu \mathrm{m}$ filaments twisted with a twist pitch of $12.7 \mathrm{~mm}$. The bare matrix dimensions of the conductor are $0.955 \mathrm{~mm}$ by $1.65 \mathrm{~mm}$. The conductor insulation is $0.025 \mathrm{~mm}$ thick. The coils are designed to be 6 layer coils that in $9.6 \mathrm{~mm}$ thick, including $2 \mathrm{~mm}$ of ground plane insulation. At an average design induction of $1.25 \mathrm{~T}$ on axis, the coil design current is about 392.9 A. The peak induction in the coil winding is about $1.6 \mathrm{~T}$, which means that the coil operating temperature margin is over $2.5 \mathrm{~K}$

It is proposed that the coils be wound and cast on a form that is removed after the coil is cured. After curing the coils are removed from the mold and machined at the ends and on the outer radial surface. After the coils are machined they can be shrunk fit into 6061 aluminum support structure that has been machined sot that the coils closely fit with in it. The 6061-aluminum support structure on the outside of coils serves the following functions; 1) It limits the coil strain by carrying some of the magnet hoop forces, and 2) it serves as a shorted secondary to protect the magnet during a quench. A single magnet is entirely selfprotecting through quench back from the support structure. One can use quench back to protect a string of these magnets as well. When one detects a quench in one magnet, the current in the string can be discharged through a varistor resistor, causing all coils to go normal through quench back from the support structure.

The longitudinal space, at the center of the magnet, available for leads, cryogenic services, and cold mass supports is about $85 \mathrm{~mm}$. How the space at the center of the magnet cryostat is utilized is illustrated in Figure 3. It is proposed that high temperature superconductor (HTS) leads be used between $40 \mathrm{~K}$ and $4.4 \mathrm{~K}$. From $40 \mathrm{~K}$ up, the leads are gas cooled using the gas flow from the shield circuit that enters the magnet system at $16 \mathrm{~K}$.

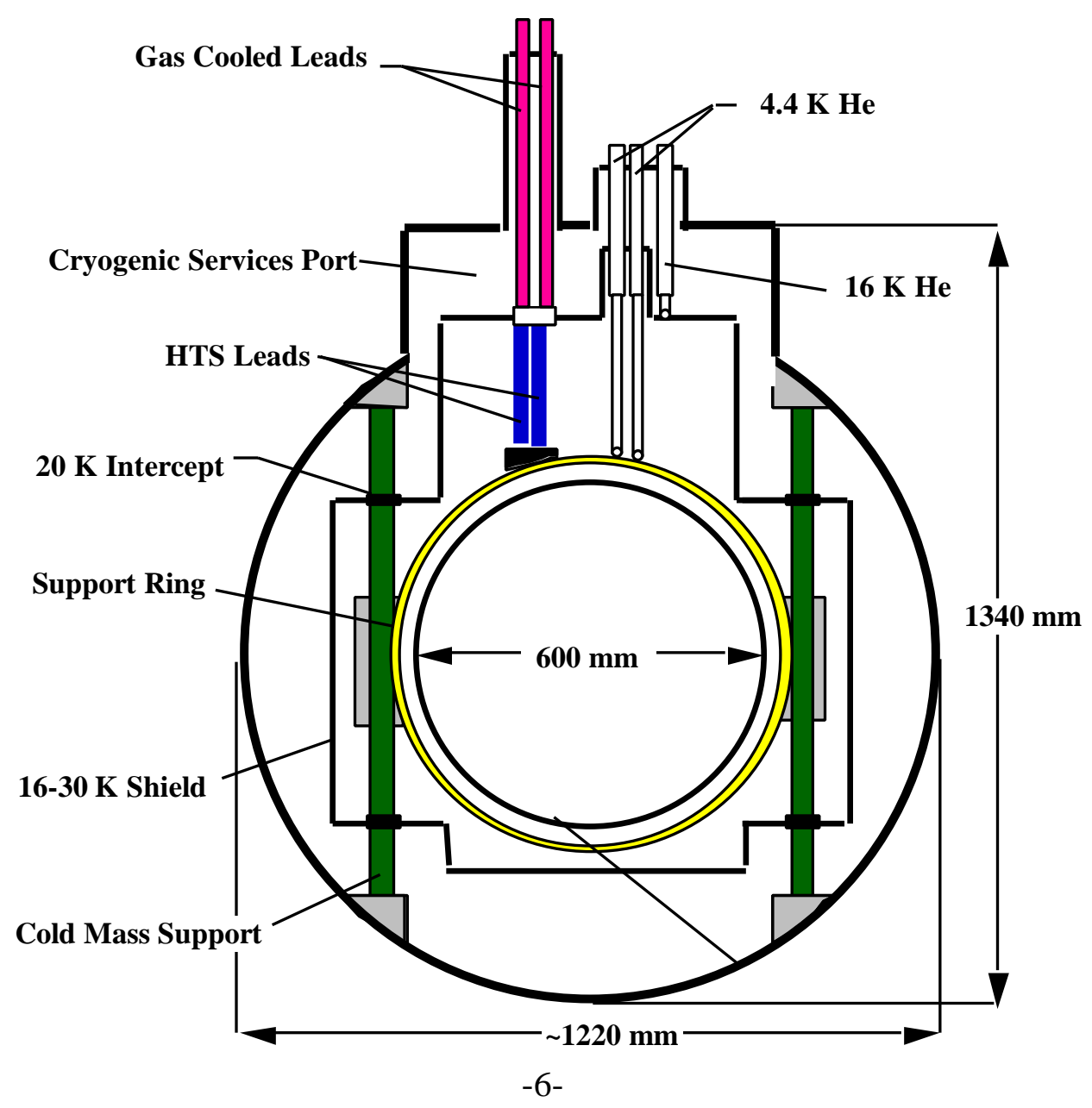


Figure 3. A Cross Section of an Induction Cell Solenoid showing the Cold Mass Supports and the Leads

The cold mass of phase-rotation solenoid (including the $30 \mathrm{~K}$ shield and lower lead assembly) is estimated to be about $210 \mathrm{~kg}$. The primary forces that will be seen between the cold mass and room temperature will be forces do to shipping and forces introduced due to unbalanced magnetic fields. The magnet cold mass supports are designed for a force of $20000 \mathrm{~N}$ in any direction. It is proposed that a pair $60 \mathrm{~mm}$ diameter oriented carbon fiber tubes (with a wall thickness of about $3 \mathrm{~mm}$ ) be used to carry forces from the cold mass to room temperature.

Since there is a solenoid magnet every meter down the phase rotation channels and the drift spaces between the phase rotation linac sections, leads must be brought out of each of these magnets. All of the magnets in 25-meter long subsets can be hooked in series and run off of common power supply. Inter-connects between the solenoids are conventional copper cable. A long string of magnets can be run from a power supply because the magnet coils are closely coupled inductively to each other and to the support structure. Quenchback is the primary mode of quench-protection for the string of magnets. When quenchback is used for quench protection all of the magnets will quench when one does. Quenchback also eliminates the forces between solenoids when one goes normal. Each one-meter long magnet section will have its own set of leads to room temperature. The leads between $4 \mathrm{~K}$ and $50 \mathrm{~K}$ will be made from high temperature superconductor (HTS). The leads from room temperature to the top of the HTS leads at $50 \mathrm{~K}$ should be gas-cooled. Gas from the refrigerator that is used to cool the magnet shields and cold mass support intercepts can be used to cool the gas-cooled leads. This gas must be returned to the refrigerator compressor intake at room temperature. See Figure 3 for a schematic representation of the cold mass support system, the helium supply system, and the current leads.

\section{MINI-COOLING SYSTEM SOLENOIDS}

The solenoids for the mini-cooler section use the same basic magnet design as the magnets used in the induction linac cells. The primary difference is the inside diameter of the superconducting coil ( $858 \mathrm{~mm}$ versus $648 \mathrm{~mm}$ for the induction cell coils). Another difference is that three coil modules (six coils) are within a single cryostat vacuum vessel. Each module has its own cold mass support system, but the three modules are hooked together using superconducting bus bars cooled with two-phase helium. There is a single set of leads powering the modules in the magnet cryostat. In the magnets that are next to the flip region, individual power supplies may be used to power the coils to shape the magnetic field in the flip region.

The solenoid for the flip channel are the same as those proposed for the decay channel except the warm bore diameter of the magnet sections is set at $800 \mathrm{~mm}$. This diameter should provide enough space for liquid the 1.75 meter long hydrogen absorbers that have window diameters of $600 \mathrm{~mm}$. This allows for a 50 to $70 \mathrm{~mm}$ space on the outside of the absorber for cooling of the hydrogen within the absorber. The two liquid hydrogen absorbers will use helium coming from the refrigerator at $16 \mathrm{~K}$ to cool them. About $9.8 \mathrm{~kW}$ of refrigeration at $16 \mathrm{~K}$ is needed to cool the absorbers. This is equivalent to about $3 \mathrm{~kW}$ of cooling at $4.4 \mathrm{~K}$. The cryogenic services to the liquid hydrogen absorber can go through the 100 -mm space between the magnet cryostats. It is also possible for these services to enter the hydrogen absorber by going through the magnet cryostat between magnet coil modules. Figure 4 shows a schematic representation of the solenoids in the mini-cooler region.

An 18-meter long decay region upstream from the first induction phase-rotation linac will have the same solenoids that are used in the mini-cooling section, except there will be 
no two-meter long field flip section within the decay channel. The $100 \mathrm{~mm}$ space between the magnet clear bore and the cryostat warm bore will be filled with a water-cooled copper radiation absorber used to reduce the ionizing radiation heating in the magnet coils.

With the exception of the two-meter long field flip solenoid all of the solenoids in the mini-cooler channel will be powered using the same power supply. All six magnets in the decay channel can be powered from a single power supply. Field correction dipoles are assumed to be mounted on the inner bore of the solenoid cols. These coils can be1-mm thick and they will correct alignment errors up to $5 \mathrm{mrad}$. 


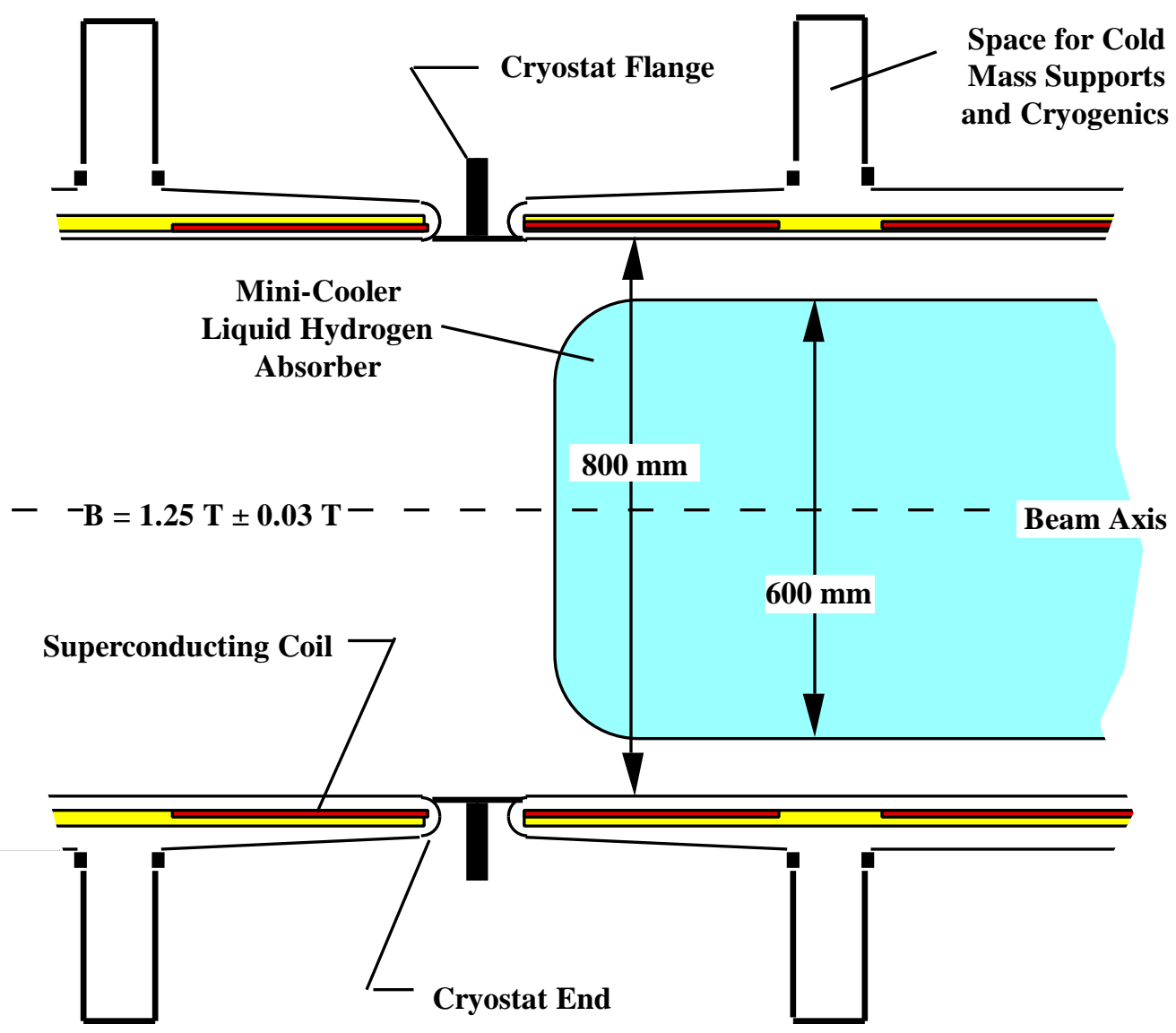

Figure 4. A Cross-section of the Solenoids for the Mini-cooler between the Induction Linac Sections

\section{CRYOGENIC COOLING OF THE PHASE ROTATION MAGNETS}

The induction cell solenoids, the decay solenoids, and the induction cell solenoids are cooled by conduction from the 6061-aluminum support structure. The aluminum support structure will be cooled by two-phase helium flowing in tubes attached to the support structure. Two-phase helium cooling is commonly used to cool large detector magnets. The advantages of two-phase tubular cooling are as follows: 1) There is a small helium inventory within the magnet. 2) The two-phase helium tubes have a high-pressure rating. This means that the magnet cryostat is not a pressure vessel. 3) Two-phase helium cooling does not require a cold compressor or a helium pump to circulate the helium through the magnet cooling system. 4) The temperature of the helium in a two-phase helium cooling circuit decreases as it moves along the flow circuit. 5) The pressure drop along a two-phase helium flow circuit is lower than for a comparable supercritical helium forced flow circuit.

It is assumed that twenty to twenty-five magnets that are cooled in series from the twophase helium refrigerator and control cryostat. If twenty to twenty-five magnets are cooled, the mass flow rate through the flow circuit should be about 2.5 grams per second. The twophase helium tube would be attached to the superconducting coil support structure, the base of the HTS leads and the attachment points of the cold mass supports. The heat loads into the magnet cryostat at $4.4 \mathrm{~K}$ and $30 \mathrm{~K}$ is shown in Table 2.

The heat that is added to the two-phase helium flow stream at $4.4 \mathrm{~K}$ in each meter of solenoid is expected to vary from $0.45 \mathrm{~W}$ to $3.0 \mathrm{~W}$ depending on the heat input due to ionizing radiation from the proton target. The peak temperature at the inside of the superconducting when the ionizing radiation heat load is highest (a maximum value of $4.5 \mathrm{~W}$ 
in the first solenoid of the first induction linac) is expected to be less than $5.5 \mathrm{~K}$. Except for heat from ionizing radiation from the target, the heat load at $4.4 \mathrm{~K}$ is dominated by the heat leak down the HTS current leads.

TABLE 2. The Sources of Heat at $4.4 \mathrm{~K}$ and $30 \mathrm{~K}$ in a 1-meter Long Induction Cell Magnets

\section{Source of Heat}

Heat Flow Down the Cold Mass Supports

Thermal Radiation through the Multi-layer Insulation

Heat Flow down the Helium Bayonet Joints

Heat Flow down Instrumentation Wires

Heat Flow down the 400 A Magnet Current Leads

Heating due to Ionizing Radiation from the Target

\section{TOTAL HEAT LOAD PER METER}

4.4 K Load (W)

0.12

0.05

0.03

0.02

0.25

0.0 to 2.5

0.47 to 3.0
$16 \mathrm{~K} \operatorname{load}(\mathrm{W})$

1.9

2.0

1.3

0.1

0 to 0.3

The heat load into the shield circuit stream is expected to vary from $5.3 \mathrm{~W}$ to $5.6 \mathrm{~W}$ depending on the heat input due to the ionizing radiation from the proton target. The shield gas comes from the refrigerator at a temperature $16 \mathrm{~K}$. (The same $16 \mathrm{~K}$ helium is used to cool the mini-cooler.) This gas enters the magnet cryostat through a single vacuum insulated tube. The helium flow in this tube is dictated by the needs of the gas-cooled leads between $50 \mathrm{~K}$ and room temperature. The 400 A leads gas cooled leads will need about 0.05 grams per second. The shield gas stream picks up heat from the cold mass supports, thermal radiation on the shield, helium bayonet joints, and the instrumentation wires. In most of the induction cells the expected heat load into this stream is about 5.3 watts per meter. In the first cells of the first induction accelerator, the shield circuit may pick up as much as $5.8 \mathrm{~W}$. (The extra $0.3 \mathrm{~W}$ is due to ionizing radiation from the target heating in the $40 \mathrm{~K}$ shields.) The helium stream temperature entering the shield circuit from the refrigerator rises from 22 to $24 \mathrm{~K}$ as it flows to the base of the gas-cooled leads. The gas used to cool the shields and the cold mass support intercepts will be used to cool the gascooled leads between about $40 \mathrm{~K}$ and room temperature. The high temperature superconductor leads (HTS) leads are designed to operate with their top end temperature below $70 \mathrm{~K}$. The gas exiting the room temperature end of the gas-cooled leads returns warm to the refrigerator compressor suction. Figure 5 shows a schematic of helium cooling system for a typical one-meter long induction-linac phase-rotation solenoid. 


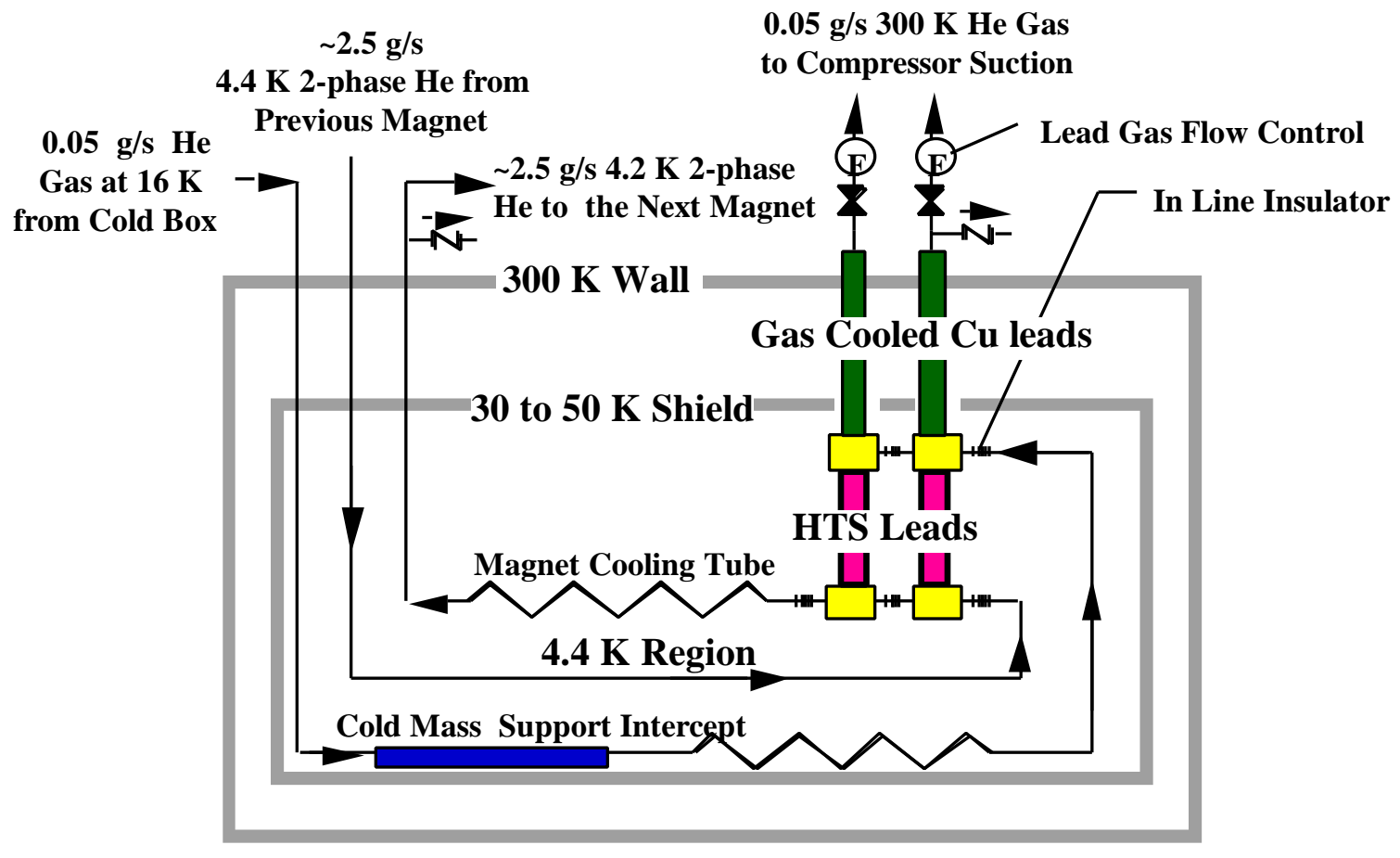

Figure 5. Cryogenic Cooling System Schematic for Cooling within a Typical Induction Cell Cryostat THE MAGNET POWER SUPPLY AND QUENCH PROTECTION

A string of twenty-five induction cell solenoids has a self-inductance of 68.5 to $72.5 \mathrm{H}$ depending on which induction linac they are in. A string of six 3-meter long solenoids for the decay channel and the mini-cooler will have a self-inductance of $85.8 \mathrm{H}$. The magnets in any of the strings can be charged to full field in less than 1800 seconds, by a power supply that delivers $500 \mathrm{~A}$ at voltages up to $50 \mathrm{~V}$. The power supply controllers should regulate on the voltage across magnet during magnet charging. Once the superconducting solenoids are charged, the current is kept constant.

Because the magnets are closely coupled, a quench in one magnet of the string will trigger quenches in all of the magnets in the string. A typical magnet quench will raise the temperature of the magnet cold mass to about $45 \mathrm{~K}$. The helium refrigerator will be sized to allow the magnet to be cooled back down to $4.4 \mathrm{~K}$ in less than two hours. To cool a string of magnets from $45 \mathrm{~K}$ to $4.4 \mathrm{~K}$ in two hours, the helium refrigeration system must deliver helium at 10 to $15 \mathrm{~K}$ to the magnets at rate of 4.5 grams per second.

\section{CONCLUDING COMMENTS}

From the studies done for this paper and for Ref. 5, it is clear that a thin superconducting solenoid can be installed within the cells of an induction linac. When the magnetic induction within the bore of the solenoid is $1.25 \mathrm{~T}$, the magnetic induction within the cores of the induction linac can be kept below $0.2 \mathrm{~T}$. The superconducting solenoid design that is proposed for the neutrino factory phase-rotation system appears to be entirely feasible. The designed concept is advanced enough to build an I-meter long prototype magnet.

Cooling can be provided by two-phase helium in tubes attached to the superconducting coil support structure. There is plenty of temperature margin in the coil to allow for it to be cooled from the support structure by conduction. The shields and gas-cooled leads can be 
cooled using gaseous helium from the refrigerator supplied to the magnet at $16 \mathrm{~K}$. The same circuit can also be used to cool the mini-cooler liquid hydrogen absorbers.

The superconducting solenoid quench protection system uses quench back from the coil support structure to protect the superconducting coils. A quench in one magnet will cause the string of magnets to go normal. There is adequate available cooling available to cool the entire phase rotation channel from $45 \mathrm{~K}$ to $4.4 \mathrm{~K}$ is less than three hours.

\section{ACKNOWLEDGEMENTS}

This work was performed at the Lawrence Berkeley National Laboratory with the support of the Office of Science, United States Department of Energy under DOE contract DE-AC03-76SF00098.

\section{REFERENCES}

1. N. Holtkamp and D. Finley eds., "A Feasibility Study of a Neutrino Source Based on a Muon Storage Ring," Fermilab-Pub-00/108E, (2000)

2. S. Ozaki, R. B. Palmer, M. S. Zisman and J. C. Gallardo eds., "Feasibility Study II of a Muon-Based Neutrino Source," BNL-52623, June 2001

3. M. A. Green, E. L. Black, R. C. Gupta, et al, "The Role of Superconductivity and Cryogenics in the Neutrino Factory," submitted to Advances in Cryogenic Engineering 47 (This Proceedings) (2001)

4. L. Reginato, S. Yu, M. Green, and D. Vanecek, "Muon Phase Rotation using an Induction Linac," to be published in the Proceedings of PAC-01, Chicago IL, 18-22 June 2001

5. "A Thin Superconducting Solenoid for Use in a Phase Rotation Induction Linac," co-authored with J. Fockler, R. E. Lafever, D. L Vanecek, and S. S. Yu, IEEE Transactions on Applied Superconductivity 11, p 2180, (2001) 


\title{
Superconducting Magnets for Induction Linac Phase-Rotation in a Neutrino Factory
}

\author{
M. A. Green, and S. Yu \\ Lawrence Berkeley National Laboratory, Berkeley CA 94720, USA
}

July 2001

Presented at the 2001 Cryogenic Engineering Conference

Madison Wisconsin, USA

17 July through 20 July 2001 
* This work was performed at the Lawrence Berkeley National Laboratory with the support of the Office of Science, United States Department of Energy under DOE contract number DE-AC03-76SF00098. 